\title{
Editorial: Trust, value systems and governance in collaborative networks
}

\author{
Luis M. Camarinha-Matos • Arturo Molina
}

Received: 2 September 2008 / Accepted: 2 September 2008 / Published online: 6 November 2008

(C) Springer Science+Business Media, LLC 2008

Various recent developments in Collaborative Networks (CNs) attempt to model a number of aspects that are characterized by an inherent imprecision and incompleteness of information. In fact, collaborative networks are complex entities whose proper understanding, design, implementation, and management require the integration of different modeling perspectives and techniques. Perspectives related to behavior and decision making in face of incomplete and imprecise information are particularly difficult to model. CNs involve a large number of autonomous and heterogeneous entities—organizations, people, systems—often with: (i) different value systems, and therefore different perceptions of value and importance/priority of things, and (ii) a behavior that is influenced by factors such as emotions, preferences, working habits, ethical values, level of trust, competences, etc. Some of these aspects have a socio-organizational and anthropocentric nature that is difficult to capture with traditional logic modeling approaches.

Complementarily, new reasoning and decision making approaches are being developed resorting to qualitative reasoning and computational intelligence methods. The aim is to offer better approaches to understand and govern collaborative networks in order to maximize the benefits and reduce the risks of these organizational forms.

This special issue comprises a selection of papers from the PRO-VE'07 conference, representing examples of relevant work in this direction. The PRO-VE conference series, spon-

L. M. Camarinha-Matos $(\varangle)$

Faculty of Sciences and Technology, New University of Lisbon,

Lisbon, Portugal

e-mail: cam@uninova.pt

A. Molina

Center of Innovation in Design and Technology, Tecnológico

de Monterrey, Monterrey, Mexico sored by IFIP (International Federation for Information Processing) and SOCOLNET (International Society of Collaborative Networks), has a recognized position as the most focused scientific/technical conference in the area, offering a major opportunity for the presentation and discussion of both the latest research developments and industrial practice case studies.

The 2007 edition, held in Guimarães, Portugal, continued a series of successful conferences started with PRO-VE'99 (held in Porto, Portugal), and continued with PRO-VE 2000 (Florianópolis, Brazil), PRO-VE'02 (Sesimbra, Portugal), PRO-VE'03 (Lugano, Switzerland), PRO-VE'04 (Toulouse, France), PRO-VE'05 (Valencia, Spain), PRO-VE'06 (Helsinki, Finland), and PRO-VE'07 (Guimarães, Portugal).

The following topics had been addressed as part of this special issue:

- Trust, which is a crucial element in collaboration. In addition to giving a general overview of the topic, the article by Msanjila and Afsarmanesh introduces a framework aimed at supporting the establishment of rational trust relationships among organizations in a virtual organizations breeding environment. It also discusses risks versus trust.

- Value systems, whose understanding is vital for the establishment of effective governance mechanisms in CNs. One article by Romero, Galeano and Molina discusses the base related concepts, giving a comprehensive perspective of the value system concept and its elements, including both the socio-economic and the ethical perspectives. The work of Camarinha-Matos and Macedo represents an attempt to formalize the concept of value system, providing a novel conceptual framework, and discussing its applicability in CNs.

- Benefits and performance measurement, which are key elements in $\mathrm{CN}$ management. The work of Cheikhrouhou, Piot and Pouly introduces a multi-criteria model for 
the evaluation of business benefits in CNs, discussing its applicability in the selection of an optimal network of partners and in the evaluation of their corresponding benefits. Westphal, Thoben and Seifert focus on collaboration performance measurement and its use in virtual organizations management.

- Legal issues and contracting in electronic markets. Conrad et al. discuss the legal challenges in electronic markets for short-lived or non-material products and introduce a platform particularly focused on distributed market places for energy.
Collaborative networks represent a young scientific discipline that requires the combination of a multiplicity of scientific backgrounds to properly address its complex challenges. Therefore, there are certainly many more challenges ahead in the topics addressed in the included papers. Nevertheless this issue is expected to represent a step in the consolidation of this new discipline and both the authors and referees are thanked for their valuable contribution. 